\title{
Estabilidade do reposicionamento anterior da maxila através de análise cefalométrica lateral
}

\author{
Rodrygo Nunes Tavares*, Luis Augusto Passeri**
}

\begin{abstract}
Resumo
Este estudo cefalométrico retrospectivo foi proposto objetivando analisar a estabilidade do reposicionamento anterior da maxila, utilizando-se a osteotomia Le Fort I. A amostra consistiu de 30 telerradiografias de dez pacientes submetidos a um avanço cirúrgico da maxila, sem segmentação desta ou associação com qualquer cirurgia na mandíbula, realizado pela Área de Cirurgia Buco-Maxilo-Facial da Faculdade de Odontologia de Piracicaba - Unicamp. A mesma técnica de fixação interna rígida foi utilizada para todos os pacientes, e nenhum enxerto ou substituto ósseo foi usado. Três radiografias cefalométricas laterais foram realizadas para cada paciente, nos períodos pré e pós-operatórios imediatos, e com, no mínimo, seis meses após a cirurgia. Sobre estas radiografias foram feitas linhas de referência horizontal e vertical. Os pontos cefalométricos utilizados para calcular as mudanças de posição da maxila foram tanto esqueléticos, quanto dentários. A análise estatística (teste t de student pareado) não demonstrou diferença estatística significante entre os intervalos pós-operatórios imediato e com no mínimo seis meses. Concluímos que o reposicionamento anterior da maxila, utilizando-se a osteotomia Le Fort I, com fixação interna rígida e sem o uso de enxertos autógenos ou qualquer substituto ósseo, é um procedimento estável.
\end{abstract}

Palavras-chave: Osteotomia Le Fort I. Estabilidade. Fixação interna rígida. Cirurgia ortognática.

\section{INTRODUÇÃO}

A osteotomia Le Fort I da maxila tem sido utilizada freqüentemente para correção das deformidades esqueléticas do terço médio da face. O seccionamento da maxila usando-se cinzéis por meio de incisões transcutâneas foi inicialmente descrito, na Europa, por Von Langenbeck, em 1859, e onze anos mais tarde na América do Norte, por Cheever (apud HOFFMAN, MOLONEY'16, 1996). Esses relatos iniciais foram realizados objetivando o tratamento de tumores. Entretanto, a osteotomia Le Fort I "moderna" foi introduzida, aproximadamente, cem anos depois, com os relatos de Obwegeser $^{26}$, em 1965 e 1969. Esses estudos revelaram que a osteotomia associada com a mobilização da maxila foi o método mais satisfatório para sobrepujar a resistência do tecido mole, favorecendo o reposicionamento da maxila.

Porém, as bases biológicas e os princípios cirúrgicos da osteotomia Le Fort I permaneceram

* Mestre em Cirurgia e Traumatologia Buco-Maxilo-Faciais pela Faculdade de Odontologia de Piracicaba - Unicamp.

Professor Substituto da Disciplina de Cirurgia Buco-Dentária da Universidade Federal do Ceará.

** Professor Adjunto da Área de Cirurgia Buco-Maxilo-Facial - Faculdade de Odontologia de Piracicaba - Unicamp. 
pouco estudados até os trabalhos clássicos de Bell. Este autor ${ }^{3}$, por meio de estudos angiográficos e histológicos, definiu todo o processo de revascularização do segmento distal da maxila, após a osteotomia Le Fort I, concluindo ser este um procedimento viável. Em estudo subseqüente, publicado no mesmo ano, Bell et al..$^{5}$ descreveram detalhadamente a técnica necessária para conseguir-se um resultado previsível e estável com essa osteotomia. Desde então, ela vem sendo adotada para o tratamento de pacientes, com má oclusão do tipo Classe III esquelética, devida à deficiência de maxila ${ }^{4}$.

Para a obtenção de sucesso, após a realização dos movimentos cirúrgicos da maxila, é necessário restaurar a função mastigatória, promover uma estética agradável e obter estabilidade a longo $\mathrm{prazo}^{4}$. O movimento pós-operatório do segmento distal da maxila osteotomizada, em direção à sua posição pré-operatória (recidiva), continua a ser um problema substancial após a osteotomia Le Fort I. Diversos fatores contribuem para o desenvolvimento da recidiva, como a qualidade do reparo ósseo, retração cicatricial, ausência de comprometimento vascular, técnica cirúrgica empregada, qualidade da Ortodontia pré e pós-cirúrgica, interferência do septo nasal durante a cirurgia, tempo de bloqueio maxilomandibular, mobilização e fixação inadequadas, uso de enxertos ou substitutos ósseos, qualidade da oclusão final ${ }^{11,28}$.

Três tipos distintos de movimentos da maxila são consistentemente descritos: os reposicionamentos superior, inferior e anterior.

Inúmeros estudos avaliaram a estabilidade do reposicionamento anterior da maxila ${ }^{1,9,11,14,16 \text {, }}$ $18,20,23,27,30,31,32$. Estes trabalhos freqüentemente apresentam grupos de pacientes heterogêneos, ou seja, um único grupo envolvendo indivíduos fissurados e não-fissurados, cirurgias segmentadas e únicas de maxila, associação ou não com cirurgias de mandíbula, utilização de diferentes métodos de fixação, o que implica em dificuldades para definir qual o motivo real da recidiva.
Existem diversas formas descritas para se evitar problemas com a recidiva após o avanço da maxila, sendo a modificação da técnica da osteotomia Le Fort I o método de fixação utilizado, o uso de enxertos ou substitutos ósseos e a associação com a osteotomia sagital bilateral dos ramos mandibulares, as mais relatadas e estudadas ${ }^{11,28}$.

Com isso, é oportuno realizar um estudo avaliando a estabilidade de um grupo de pacientes submetidos a avanços da maxila, sob certas condições pré-estabelecidas, de forma a verificar se estas são suficientes para promover estabilidade.

\section{MATERIAIS E MÉTODOS Seleção da amostra}

Depois de aprovado pelo Comitê de Ética da Faculdade e Odontologia de Piracacicaba - Unicamp, sob o protocolo número 094/2001, foram selecionadas 30 telerradiografias tomadas em norma lateral, padronizadas de acordo com a técnica clássica de posicionamento de Broabent ${ }^{8}$. Essas radiografias foram realizadas nos períodos pré-operatório imediato, pós-operatório imediato e pósoperatório tardio, com no mínimo 6 meses, de 10 pacientes dos arquivos da Área de Cirurgia BucoMaxilo-Facial, da mesma Faculdade supracitada, sem distinção de raça, gênero ou cor.

Como critérios para inclusão neste estudo, o paciente necessitava apresentar, no mínimo, três telerradiografias, sendo uma em cada período supracitado. Não poderia apresentar fissura lábiopalatina. Todos eram adultos com crescimento já finalizado. Em nenhum caso foi utilizado enxerto ósseo interposicional e não houve associação com osteotomias mandibulares. Todos os pacientes foram submetidos ao preparo ortodôntico antes da realização da cirurgia. A técnica cirúrgica empregada foi semelhante à descrita por Bell et al. ${ }^{3}$ Como método de fixação óssea da maxila foram utilizadas quatro placas do sistema 1,5mm (Synthes ${ }^{\mathrm{TM}}$ ). Sendo uma placa em forma de "L", de quatro furos, fixada na região de pilar nasomaxilar, adjacente à abertura piriforme, e uma placa reta, 
de dois furos, na região de pilar zigomaticomaxilar, em ambos os lados. Os parafusos utilizados foram todos do tipo autoroscáveis, de 6 ou $8 \mathrm{~mm}$ de comprimento. Não se utilizou bloqueio maxilomandibular pós-operatório por nenhum periodo neste estudo.

\section{Traçado cefalométrico}

As telerradiografias, tomadas em norma lateral, foram realizadas seguindo a técnica padrão

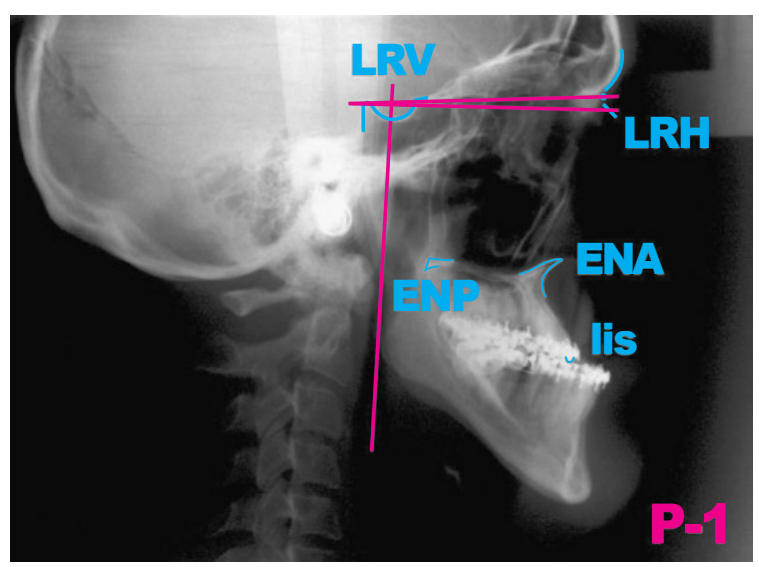

FIGURA 1 - Traçado P-1.

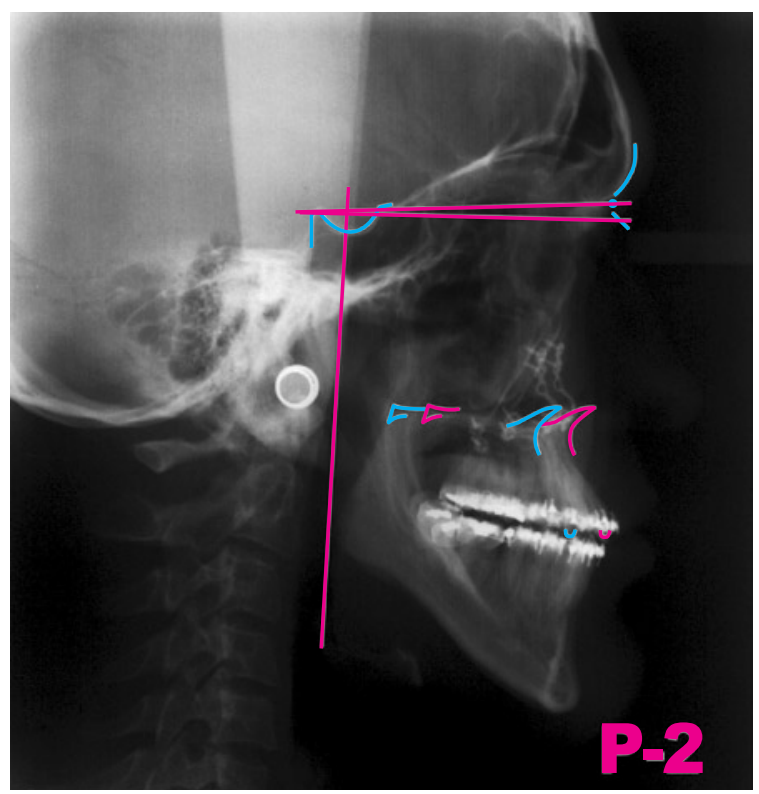

FIGURA 2 - Traçado P-2. para análise das estruturas da face. Dessa forma as radiografias foram padronizadas com o paciente posicionado no cefalostato, mantendo os lábios em repouso e máxima intercuspidação habitual, obtidas de acordo com a técnica de posicionamento proposta por Broadbent ${ }^{8}$.

Sobre as radiografias foram adaptadas folhas de papel acetato (Microimage 4000) - Film laser de $18 \times 24 \mathrm{~cm}$ e espessura de $0,07 \mathrm{~mm}$, com o auxílio de fita adesiva (Scotch $3 \mathrm{M}$ ) de $11 \mathrm{~mm}$. Foram colocadas sobre um negatoscópio com duas lâmpadas fluorescente, brancas, de mesmo tamanho e eqüidistantes. Para uma melhor visualização das radiografias, os traçados foram realizados em uma sala escura. Para delineamento das estruturas anatômicas utilizou-se uma lapiseira (Calno SH-GC $0,5 \mathrm{~mm}$ ) com grafite (Uni $2 \mathrm{~B}$ de $0,5 \mathrm{~mm}$ ), régua milimetrada (Desetec modelo 7130 - Trident), transferidor (Desetec $n^{\circ}$ 8115), borracha (Carbex 40/20) e para delineamento dentário (Tracing Template - Orthocontics). As mensurações foram realizadas, manualmente, com o auxílio da mesma régua milimetrada.

Os cefalogramas foram confeccionados através de uma técnica de transferência de estruturas, de forma a minimizar erros de reprodutibilidade das estruturas cefalométricas de referência. Foi utilizado um traçado cefalométrico, de acordo com os estudos de Radney, Jacobs ${ }^{29}$; Ayoub et al. ${ }^{2}$;

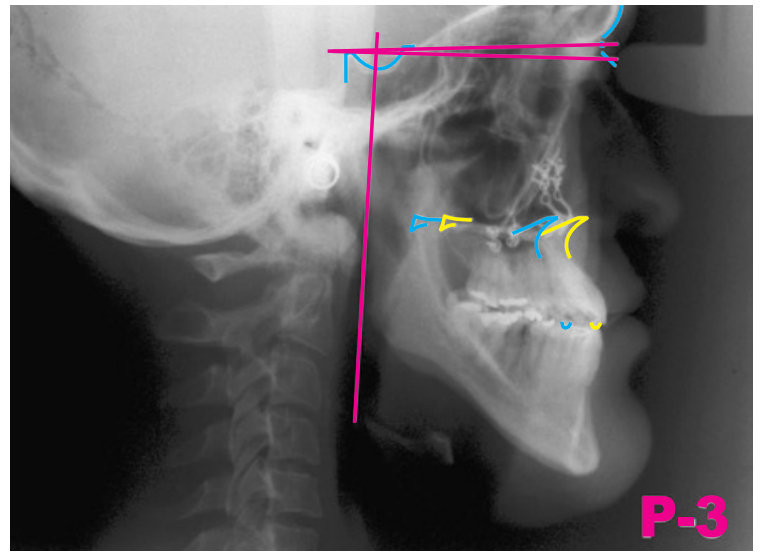

FIGURA 3 - Traçado P-3. 
Jensen et al. ${ }^{19}$ e Coelho ${ }^{10}$, padronizando duas linhas de referência, uma horizontal e outra vertical, descritas abaixo e ilustradas na figura 1 .

Os pontos e linhas cefalométricas que foram utilizados são descritos a seguir:

- Iis: Incisal do incisivo central;

- ENA: Espinha nasal anterior (ponto mais anterior da espinha nasal anterior);

- ENP: Espinha nasal posterior (ponto mais posterior da espinha nasal posterior);

- S: Ponto sela (centro geométrico da sela túrcica);

- N: Ponto násio (ponto de encontro entre a sutura do osso frontal com os ossos próprios do nariz);

- Linha SN: Linha que passa pelos pontos S e N;

- LHR: Linha horizontal de referência (traçada 12 graus abaixo da linha $\mathrm{SN}$ );

- LVR: Linha vertical de referência (traçada perpendicular à linha horizontal de referência, partindo do ponto S).

A linha horizontal de referência foi definida como sendo tomada a $12^{\circ}$ abaixo da linha SN, passando pelo ponto sela. Esta linha de referência torna-se paralela ao plano horizontal natural. A linha vertical de referência foi definida como perpendicular à linha horizontal de referência, passando por sela, conforme descrito por Coelho ${ }^{10}$.

Sobre a telerradiografia pré-operatória imediata, foram identificados a porção anterior e média da base do crânio, o osso frontal, os ossos próprios do nariz e a porção mais posterior e anterior da maxila. A partir dessas estruturas, a linha $\mathrm{SN}$ e as linhas de referências horizontal e vertical foram traçadas. Os pontos ENA, ENP e Iis foram delineados com grafite cor preta, dando origem ao primeiro traçado P-1.

O P-1 foi, então, colocado sobre a radiografia pós-operatória imediata, de modo que as estruturas de referência coincidam, ou seja, as porções anterior e média da base do crânio e os ossos frontal e próprio do nariz. Utilizando-se grafite cor verde, os pontos ENA, ENP e lis foram identificados em suas novas posições (P-2). Utilizando-se o P-2, as distâncias foram medidas dos pontos demarcado no pré e pós-operatório para as linhas de referências, utilizando-se a régua milimetrada.

Realizou-se, então, outro P-1. Este novo traçado foi posicionado sobre a radiografia pós-operatória tardia, e os mesmos pontos (Iis, ENA e ENP) foram traçados com grafite cor vermelha. As distâncias foram medidas dos pontos para as linhas de referências já existentes, utilizando-se a régua milimetrada, originando um outro traçado denominado P-3.

Dessa forma, medidas lineares horizontais foram obtidas, perpendicularmente, para cada ponto considerado nos diferentes tempos operatórios (P-2 e P-3), até as linhas de referência horizontal e vertical. Cada traçado foi repetido quatro vezes de forma a minimizar a possibilidade de erros de reprodutibilidade dos pontos cefalométricos, conforme descrito por Martins ${ }^{24}$. As médias dessas mensurações foram utilizadas para comparar os períodos pós-operatório imediato e tardio.

\section{Análise estatística}

Os dados foram tabulados no programa Biostat®, versão 1998, compatível com Windows 95, de autoria de Manuel Ayres e Manuel Ayres Junior. $O$ teste $t$ de Student, paramétrico, foi utilizado usando amostras pareadas (pós-operatório imediato e pós-operatório tardio), com o objetivo de verificar se estas medidas apresentavam diferenças significativas. O nível de significância foi de 5\%, perfazendo um nível de confiança de $95 \%$.

\section{Análise do erro de método}

A reprodutibilidade das medidas foi determinada repetindo os pontos quatro vezes (realizado pelo mesmo observador). O erro deste método foi calculado utilizando-se o teste $t$ de Student, pareado, para uma amostra, com nível de significância de $10 \%$, como recomendado por Houston ${ }^{17}$. 


\section{RESULTADOS}

Os pacientes foram submetidos a avanços que variaram desde $2,25 \mathrm{~mm}$ até $10,12 \mathrm{~mm}$, com média de $5,89 \mathrm{~mm}$ (Tab. 1). A referência utilizada para medir os avanços maxilares foi a incisal do incisivo central superior, visto ser este ponto o mais reprodutível e não sofrer alteração entre o pré-operatório e pós-operatório imediatos, segundo Louis et al. ${ }^{22}$; Luyk e Ward-Booth ${ }^{23}$.

Foram realizadas, então, as medidas de cada variável, para cada período (pré-operatório 1, pósoperatório imediato, pré-operatório 2 e pós-operatório tardio), em relação às linhas horizontal e vertical de referência. As tabelas de 2 a 7 mostram as médias das medidas realizadas dos pontos cefalométricos (ENA, ENP e Iis) às linhas de referências horizontal (LRH) e vertical (LRV), em cada periodo estipulado. $\mathrm{O}$ primeiro pré-operatório realizado no primeiro P-1 foi denominado préoperatório 1, e o pré-operatório identificado no segundo P-1 foi chamado de pré-operatório 2.

Essas medidas foram submetidas ao teste $t$ de Student, paramétrico, com amostras vinculadas (pré-operatório 1, pré-operatório 2, pós-operatório imediato e pós-operatório tardio), objetivando, inicialmente, verificar se as medidas encontradas nos pré-operatórios dos dois traçados (P-2 e P3) apresentavam diferença significativa. Logo em seguida, verificou se as medidas encontradas nos períodos pós-operatório imediato e tardio apresentavam diferenças significantes, para, com isso, avaliar a estabilidade do movimento de avanço da maxila.

Admitindo-se um nível de significância de 5\% $(\mathrm{p}=0,05)$, para que os resultados fossem estatisticamente significantes deveriam apresentar valores

Tabela 1 - Médias das mensurações lineares da incisal do incisivo superior (lis) até a linha de referência vertical (LRV), em milímetros, obtidas nas radiografias pré-operatória e pós-imediata.

\begin{tabular}{cc|} 
PACIENTES & AVANÇOS (em mm) \\
1 & 4,25 \\
2 & 2,25 \\
3 & 4,50 \\
4 & 3,50 \\
5 & 3,12 \\
6 & 5,25 \\
7 & 9,67 \\
8 & 10,12 \\
9 & 7,00 \\
10 & 9,25 \\
\hline
\end{tabular}

\begin{tabular}{|c|c|c|c|c|}
\hline PACIENTES & Pré-operatório 1 (em mm) & Pré-operatório 2 (em mm) & Pós-operatório imediato (em mm) & Pós-operatório tardio (em mm) \\
\hline 1 & 82,00 & 81,50 & 82,37 & 81,50 \\
\hline 2 & 74,87 & 74,75 & 76,37 & 75,00 \\
\hline 3 & 73,62 & 73,62 & 78,50 & 76,75 \\
\hline 4 & 70,62 & 70,00 & 77,50 & 73,00 \\
\hline 5 & 85,62 & 85,12 & 83,12 & 82,75 \\
\hline 6 & 77,62 & 77,12 & 78,00 & 79,00 \\
\hline 7 & 79,62 & 79,75 & 81,87 & 84,50 \\
\hline 8 & 76,37 & 78,75 & 83,87 & 85,37 \\
\hline 9 & 82,50 & 82,12 & 87,62 & 84,62 \\
\hline 10 & 80,62 & 81,00 & 88,37 & 86,50 \\
\hline
\end{tabular}


Tabela 3 - Médias das mensurações lineares da espinha nasal posterior (ENP) até a linha de referência horizontal (LRH), em milímetros, obtidas nas radiografias pré-operatória, pós-imediata e pós-tardia.

\begin{tabular}{|c|c|c|c|c|}
\hline PACIENTES & Pré-operatório 1 (em mm) & Pré-operatório 2 (em mm) & Pós-operatório imediato (em mm) & Pós-operatório tardio (em mm) \\
\hline 1 & 23,12 & 23,12 & 26,00 & 25,87 \\
\hline 2 & 27,75 & 27,00 & 28,62 & 29,87 \\
\hline 3 & 20,37 & 20,12 & 24,87 & 22,87 \\
\hline 4 & 14,87 & 15,50 & 24,50 & 20,62 \\
\hline 5 & 24,50 & 24,75 & 27,62 & 29,50 \\
\hline 7 & 19,37 & 19,37 & 25,50 & 23,00 \\
\hline 8 & 29,00 & 29,00 & 37,75 & 38,50 \\
\hline 10 & 20,50 & 21,50 & 30,00 & 31,50 \\
\hline
\end{tabular}

Tabela 4 - Médias das mensurações lineares da incisal do incisivo central superior (lis) até a linha de referência horizontal (LRH), em milímetros, obtidas nas radiografias pré-operatória, pós-imediata e pós-tardia.

\begin{tabular}{|c|c|c|c|c|}
\hline PACIENTES & Pré-operatório 1 (em mm) & Pré-operatório 2 (em mm) & Pós-operatório imediato (em mm) & Pós-operatório tardio (em mm) \\
\hline 1 & 83,25 & 83,00 & 87,50 & 86,50 \\
\hline 2 & 79,25 & 79,00 & 81,50 & 80,60 \\
\hline 3 & 78,00 & 78,00 & 82,50 & 81,75 \\
\hline 4 & 67,25 & 67,00 & 70,75 & 71,37 \\
\hline 5 & 81,75 & 82,25 & 84,87 & 84,25 \\
\hline 7 & 71,75 & 71,25 & 76,00 & 76,75 \\
\hline 9 & 81,62 & 81,62 & 91,30 & 89,25 \\
\hline 10 & 75,50 & 77,87 & 85,62 & 88,50 \\
\hline
\end{tabular}

Tabela 5 - Médias das mensurações lineares da espinha nasal anterior (ENA) até a linha de referência vertical (LRV), em milímetros, obtidas nas radiografias pré-operatória, pós imediata e pós-tardia.

\begin{tabular}{|c|c|c|c|c|}
\hline PACIENTES & Pré-operatório $\mathbf{1}(\mathbf{e m ~ m m})$ & Pré-operatório 2 (em mm) & Pós-operatório imediato (em mm) & Pós-operatório tardio (em mm) \\
\hline 1 & 42,00 & 42,25 & 43,50 & 42,75 \\
\hline 2 & 34,25 & 34,37 & 34,50 & 33,87 \\
\hline 3 & 43,62 & 43,62 & 43,50 & 42,12 \\
\hline 4 & 44,87 & 45,00 & 48,00 & 46,87 \\
\hline 5 & 45,12 & 45,00 & 44,12 & 42,62 \\
\hline 7 & 40,25 & 41,87 & 38,50 & 38,87 \\
\hline 9 & 42,87 & 42,87 & 44,12 & 41,37 \\
\hline 10 & 36,12 & 35,37 & 34,62 & 32,87 \\
\hline
\end{tabular}


Tabela 6 - Médias das mensurações lineares da espinha nasal posterior (ENP) até a linha de referência vertical (LRV), em milímetros, obtidas nas radiografias pré-operatória, pós imediata e pós-tardia.

\begin{tabular}{|c|c|c|c|c|}
\hline PACIENTES & Pré-operatório 1 (em mm) & Pré-operatório 2 (em mm) & Pós-operatório imediato (em mm) & Pós-operatório tardio (em mm) \\
\hline 1 & 43,75 & 43,37 & 41,37 & 42,00 \\
\hline 2 & 41,50 & 41,75 & 38,75 & 41,12 \\
\hline 3 & 46,75 & 45,75 & 45,25 & 43,62 \\
\hline 4 & 47,00 & 47,00 & 43,75 & 46,50 \\
\hline 5 & 56,62 & 56,25 & 55,37 & 55,37 \\
\hline 7 & 48,12 & 48,87 & 44,00 & 48,12 \\
\hline 9 & 51,75 & 51,75 & 49,25 & 49,87 \\
\hline 10 & 51,87 & 46,00 & 48,62 & 47,62 \\
\hline
\end{tabular}

\begin{tabular}{|c|c|c|c|c|}
\hline PACIENTES & Pré-operatório 1 (em mm) & Pré-operatório 2 (em mm) & Pós-operatório imediato (em mm) & Pós-operatório tardio (em mm) \\
\hline 1 & 69,62 & 70,12 & 69,62 & 70,75 \\
\hline 2 & 57,75 & 58,00 & 58,37 & 57,50 \\
\hline 3 & 74,87 & 74,87 & 75,37 & 72,87 \\
\hline 4 & 74,37 & 74,50 & 77,50 & 78,75 \\
\hline 5 & 77,00 & 76,62 & 76,25 & 75,25 \\
\hline 6 & 71,75 & 73,25 & 72,00 & 73,25 \\
\hline 7 & 72,87 & 73,12 & 70,25 & 63,75 \\
\hline 8 & 70,50 & 69,50 & 67,12 & 66,25 \\
\hline 9 & 67,62 & 68,25 & 67,25 & 68,00 \\
\hline 10 & 77,37 & 77,12 & 74,62 & 73,87 \\
\hline
\end{tabular}

de p>0,05. Após o cálculo estatístico, todas as médias apresentaram-se com resultados estatisticamente não-significantes.

\section{DISCUSSÃO DOS RESULTADOS}

A análise cefalométrica é utilizada com freqüência para se avaliar a estabilidade após tratamentos orto-cirúrgicos. Inúmeros pontos cefalométricos podem ser utilizados para tal fim, como o ponto A, espinha nasal anterior, espinha nasal posterior, ápice radicular do incisivo central superior, a incisal do incisivo central superior ou prosthion $^{13,15,16,22,23,30}$. Neste estudo foram utilizados a incisal do incisivo central superior e as espinhas nasais anterior e posterior, visto que apresentam excelente reprodutibilidade, como afirmam Midtgard et al. ${ }^{25}$.

Para a obtenção das medidas horizontais e verticais destes pontos cefalométricos, foi utilizado o método descrito por Radney, Jacobs ${ }^{29}$; Ayoub et al. ${ }^{2}$; Jensen et al. ${ }^{19}$ e Coelho ${ }^{10}$. A confecção das linhas de referência horizontal e vertical permite que as mensurações sejam realizadas em relação a referências fixas e reproduzíveis.

O tempo de observação pós-operatório, de no mínimo seis meses, foi utilizado no sentido de 
podermos avaliar a quantidade precisa do movimento de recidiva esquelética, visto que, se houver qualquer movimento após esse prazo, a intensidade desse fenômeno será desprezível, segundo Proffit et al. ${ }^{27}$ e Louis et al. ${ }^{22}$. Entretanto, considerando que alguns pacientes ainda se encontravam em tratamento ortodôntico, os valores relativos a medidas com referências dentais, isto é, baseadas no incisivo central superior, podem ser afetados por este fator, nestes pacientes. Da mesma forma, podemos afirmar que as medidas ósseas não sofrem mais influências deste tipo, já que, nesta etapa, desde a quarta semana pós-operatória, os pacientes não fazem mais uso de elásticos Classe III, que poderiam funcionar como contenção, limitando a recidiva neste momento.

A restauração normal da função mastigatória obtenção de estética facial e estabilidade a longo prazo são fatores fundamentais para o sucesso após $\mathrm{o}$ avanço de maxila ${ }^{4}$. Inúmeros fatores influenciam na estabilidade após este avanço, como a qualidade do reparo ósseo, retração cicatricial, ausência de comprometimento vascular, a técnica cirúrgica empregada, qualidade da Ortodontia pré e pós-cirúrgica, interferência do septo nasal, mobilização e fixação inadequadas, qualidade da oclusão final e posicionamento da maxila não-passiva.

Com o objetivo de promover melhor estabilidade após o avanço de maxila, as modificações na técnica Le Fort I ou no método de fixação e a utilização de enxertos ou implantes alógenos são as medidas mais empregadas ${ }^{22,28,30}$.

A técnica empregada neste estudo foi a descrita por Bell ${ }^{5}$. Diversos estudos demonstram estabilidade satisfatória após avanços de maxila associada com esta técnica, como Araujo et al. ${ }^{1}$; Luyk, WardBooth $^{23}$; Iannetti et al. ${ }^{18}$; Proffit et al. ${ }^{27}$; Hofman e Moloney ${ }^{16}$. Kaminishi et al. ${ }^{20}$ descreveram uma modificação no desenho da osteotomia Le Fort I, objetivando uma melhor estabilidade. Nenhum resultado clínico foi apresentado neste artigo. Há poucos relatos utilizando tal técnica, Louis et al. ${ }^{22}$ encontraram estabilidade satisfatória em um gru- po de 20 pacientes. Porém, após 3 anos, o mesmo grupo relatou outro estudo semelhante, no qual não citaram mais o uso desta modificação da técnica ${ }^{30}$.

Há uma intensa discussão em relação a qual método de fixação óssea promove uma melhor estabilidade após o avanço da maxila. Carlotti, Schendel ${ }^{9}$; Iannetti ${ }^{18}$ e Proffit et al. ${ }^{27}$ relataram resultados satisfatórios utilizando o método de fixação não-rígido. Porém, nos estudos mais recentes, os autores preferem a fixação interna rígida ${ }^{16,22,23,30}$. Eles citam como vantagens deste método um índice de recidiva estatisticamente insignificante e um melhor conforto pós-operatório. No nosso estudo foram utilizados 4 placas, do sistema $1,5 \mathrm{~mm}$, sem associação com qualquer período de bloqueio maxilomandibular pós-operatório, que demonstraram estabilidade satisfatória. Estas placas foram dispostas de forma clássica, com duas na região de pilar nasomaxilar e duas no pilar zigomaticomaxilar, sendo estas últimas com apenas dois furos, o que é uma modificação utilizada em nossa faculdade.

Outro ponto bastante controverso em relação à estabilidade após o avanço da maxila é o uso ou não de enxerto ou substituto ósseo. Nesse estudo não foi utilizado nenhum enxerto autógeno ou substituto ósseo. Esta conduta foi realizada devido às desvantagens associadas ao emprego destes, como morbidade acentuada e o maior potencial de infecção, além da incerteza em relação à efetividade desta alternativa quanto à melhora na estabilidade ${ }^{16,22,23,32}$.

A quantidade de movimento anterior que a maxila é submetida pode influir na sua estabilidade pós-operatória. Segundo diversos autores, em movimentos maiores que $6 \mathrm{~mm}$, a quantidade de recidiva torna-se maior ${ }^{1,12,22,27,30}$. No nosso estudo, há uma grande variação nesta quantidade, desde avanços menores que $3 \mathrm{~mm}$ até aqueles maiores que $10 \mathrm{~mm}$. Com isso, podemos dividir nosso grupo de pacientes em dois subgrupos, segundo a quantidade maior $(n=4)$ ou menor que $6 \mathrm{~mm}(\mathrm{n}=6)$. 
Avaliando-se estes dois subgrupos, não foi encontrada diferença estatística significante entre eles, nos períodos pós-operatório imediato e tardio, em relação a nenhum ponto cefalométrico.

Outra questão abordada na literatura é o papel da osteotomia sagital bilateral dos ramos mandibulares na estabilidade da maxila após o avanço. Não há consenso se esta associação é benéfica ou prejudicial à estabilidade da maxila. Autores como La Banc et al. ${ }^{21}$ e Iannetti et al. ${ }^{18}$ afirmam que a osteotomia mandibular diminui a estabilidade maxilar. Já Brammer et al. ${ }^{7}$ e Hoffman et al. ${ }^{15}$ concluíram que ocorre exatamente o oposto, com uma melhora na estabilidade. Há, também, aqueles que sustentam que as cirurgias na mandíbula não provocam nenhum efeito na estabilidade da maxila, como Bothur et al. ${ }^{6}$. Diante dessa incerteza, no nosso estudo, nenhum paciente avaliado foi submetido à osteotomia sagital bilateral dos ramos mandibulares.

\section{CONCLUSÕES}

Com base nos resultados obtidos, pode-se concluir que:

1) O reposicionamento anterior da maxila, utilizando-se a técnica da osteotomia Le Fort I, com fixação interna rígida e sem o uso de enxertos autógenos ou qualquer substituto ósseo, é um procedimento estatisticamente estável.

2) O método de fixação interna rígida utilizando-se duas placas em "L" na região de pilar nasomaxilar, com dois parafusos por segmento, e duas placas retas, no pilar zigomático-maxilar, com um parafuso por segmento, do sistema $1,5 \mathrm{~mm}$, é um método que promove estabilidade satisfatória após o avanço de maxila.

\section{Lateral cephalometric analysis of the stability of maxillary anterior repositioning}

\footnotetext{
Abstract

This is a retrospective cephalometric study designed to investigate the stability after anterior maxilla repositioning, by Le Fort I osteotomy. Thirty cephalometric radiographs were selected, from ten patients submitted to one-piece surgical maxillary advancement, with no concomitant mandibular surgery, performed by the Division of Oral and Maxillofacial Surgery of Piracicaba Dental School - Unicamp. These cases were submitted to the same internal rigid fixation technique, with no use of bone grafts or any bone substitute. Three lateral cephalometric radiographs were obtained for each patient, immediately presurgery and postsurgery, and at least six months after surgery. Lines were draw upon the X-rays to serve as horizontal and vertical reference lines. The landmarks used to measure maxillary positional changes consisted of skeletal and dental features. Statistical analysis (paired t student test) showed no significant differences between the immediately postsurgery and at least six months postsurgery periods. We concluded that the anterior maxillary repositioning by Le Fort I osteotomy, using rigid internal fixation, with no bone grafts or any bone substitute, is a stable procedure.

Key words: Le Fort I osteotomy. Stability. Internal rigid fixation. Orthognathic surgery.
} 


\section{REFERÊNCIAS}

1. ARAUJO, A. et al. Total maxillary advancement with and without bone grafting. J Oral Surg, Chicago, v. 36, no.11, p. 849-858, 1978.

2. AYOUB, A.F. et al. Soft tissue response to anterior maxillary osteotomy. Int J Adult Orthodon Orthognath Surg, Chicago, v. 6, no. 3, p.183-190, 1991.

3. BELL, W. H. et al. Bone healing and revascularization after total maxillary osteotomy. J Oral Surg, Chicago, v. 33, no. 4, p. 253260, 1975.

4. BELL, W. H. et al. Simultaneous repositioning of the maxilla, mandible and chin. Am J Orthod, St. Louis, v. 89 no.1, p. 28-50, 1986.

5. BELL, W. H. Le Fort I osteotomy for correction of maxillary deformities. J Oral Surg, Chicago, v. 33, no. 6, p. 412-426, 1975.

6. BOTHUR, S. et al. Stability of Le Fort I osteotomy with advancement: a comparison of single maxillary and two-jaw procedure. J Oral Maxillofac, Philadelphia, v. 56, no. 9, p.1029-1033, 1998.

7. BRAMMER, J. et al. Stability after bimaxillary surgery to correct maxillary excess and mandibular deficiency. J Oral Surg, Chicago, v. 38, no. 9, p. 664-670, 1980.

8. BROADBENT, B. H. A new technique and its application to Orthodontia. Angle Orthod, Appleton, v.1, no. 2, p. 45-66, 1931.

9. CARLOTTI, A. E.; SCHENDEL, S. A. An analysis of factors influencing stability of surgical advancement of the maxilla by the Le Fort I osteotomy. J Oral Maxillofac Surg, Philadelphia, v. 45, no. 11 , p. 924-928, 1987.

10. COELHO, U. Efeito da sutura em duplo V-Y, associada à sutura das bases alares, sobre o lábio superior, após o avanço da maxila: estudo cefalométrico comparativo. 1995. $110 \mathrm{f}$. Dissertação (Mestrado)-Faculdade de Odontologia de Araraquara, Unesp, 1995.

11. COSTA, F. et al. Stability of Le Fort I osteotomy in maxillary advancement: review of the literature. Int J Adult Orthodon Orthognath Surg, Chicago, v. 14, no. 3, 207-213, 1999

12. COSTA, F. et al. Stability of skeletal Class III malocclusion after combined maxillary and mandibular procedures. Int J Adult Orthod Orthognath Surg, Chicago, v. 16, no. 3, p.179-192, 2001.

13. EGBERT, M. et al. Stability of Le Fort I osteotomy with maxillary advancement: a comparison of combined wire fixation and rigid fixation. J Oral Maxillofac Surg, Philadelphia, v. 53, no. 3, p. 243-248, 1995.

14. EPKER, B. N.; SCHENDEL, S. A. Total maxillary surgery. Int J Oral Surg, Copenhagen, v. 9, no.1, p.1-24, 1980.

15. HOFFMAN, G. R. et al. The stability of facial advancement surgery (in the manegement of combined mid and lower dentofacial deficiency). J Craniomaxillofac Surg, Edindurgh, v.22, no. 2, p. 86-94, 1994.

16. HOFFMAN, G. R.; MOLONEY, F. B. The stability of facial osteotomies. Part 5. Maxillary advancement with miniplate and screw fixation. Aus Dent J, Sydney, v. 41, no.1, p. 21-27, 1996.

17. HOUSTON, W. J. B. The analysis of errors in orthodontic measurement. Am J Orthod, St. Louis, v. 82, no. 4, p. 382-390, 1983.
18. IANNETTI, G. et al. Five-year follow-up of the Le Fort I osteotomies. J Craniomaxillofac Surg, Stuttgart, v. 15, no. v. 5, p. 238-243, 1987.

19. JENSEN, A. C. et al. Soft tissue changes associated with double jaw surgery. Am J Orthod Dentofacial Orthop, St. Louis, v.101, no. 3, p. 266-275, 1992.

20. KAMINISH, R. W. et al. Improved maxillary stability with modified Le Fort I technique. J Oral Maxillofac Surg, Philadelphia, v. 41 , no. 3, p. 203-205, 1983.

21. $L A B A N C$, J. P. et al. Results following simultaneous mobilization of the maxilla and mandible for the correction of dentofacial deformities: analysis of 100 consecutive patients. Oral Surg Oral Med Oral Pathol, St. Louis, v. 54, no. 6, p. 607-612, 1982.

22. LOUIS, P. J. et al. Long-term skeletal stability after internal rigid fixation of Le Fort I osteotomies with advancements. Int J Oral Maxillofac Surg, Copenhagen, v. 22, no. 2, p. 82-86, 1993.

23. LUYK, N. H.; WARD-BOOTH, R. P. The stability of the Le Fort I osteotomies using bone plates without bone grafts. J Oral Maxillofac Surg, Philadelphia, v.13, no. 6, p. 250-253, 1985.

24. MARTINS, L. P. Erro de reprodutibilidade das medidas das análises cefalométricas de Steiner e de Ricketts pelos métodos convencional e computadorizado. 1993. Dissertação (Mestrado)- Faculdade de Odontologia de Araraquara, Unesp, Araraquara, 1993.

25. MIDTGARD, J. et al. Reproducibility of cephalometric landmarks and errors of measurements of cephalometric cranial distances. Am J Orthod, St. Louis, v. 44, no. 1, p. 56-61, 1974.

26. OBWEGESER, H. Surgical correction of small or retropositioned maxillae: the "dish-face" deformity. Plast Reconstr Surg, Baltimore, v. 43, no. 4, p. 351-365, 1969.

27. PROFFIT, W. R. et al. Stability after surgical-orthodontic correction of skeletal class III malocclusion. II Maxillary advancement. Int J Adult Orthodon Orthognath Surg, Chicago, v. 6, no. 2, p. 71-80, 1991.

28. PROFFIT, W. R.; TURVEY, T. A.; PHILLIPS, C. Orthognathic surgery: a hierarchy of stablity. Int J Adult Orthod Orthognath Surg, Chicago, v. 11, no. 3, p.191-204, 1996.

29. RADNEY, L. J.; JACOBS, J. D. Soft tissue changes associated with surgical total maxillary intrusion. Am J Orthod, St. Louis, v. 80, no. 2, p. 191-212, 1981.

30. WAITE, P. D. et al. The stability of maxillary advancement using Le Fort I osteotomy with and without genial bone grafting. Int J Oral Maxillofac Surg, Copenhagen, v. 25, no. 4, p. 264-267, 1996.

31. WARDROP, R. W.; WOLFORD, L. M. Maxillary stability following downgraft and/or advancement procedures with stabilization using rigid fixation and porous hydroxylapatite implants. J Oral Maxillofac Surg, Philadelphia, v. 47, no. 4, p. 336-342, 1989.

32. WILLMAR, K. On Le fort I osteotomy:a follow-up study of 106 operated patients with maxillo-facial deformity. Scand J Plast Reconst Surg, Stockolm, v.12, p.1-68, 1974. Supplement, 12.

\author{
Endereço para correspondência \\ Luis Augusto Passeri \\ Área de Cirurgia Buco-Maxilo-Facial \\ Faculdade de Odontologia de Piracicaba - Unicamp \\ Av. Limeira, 901 \\ CEP: 13414-903 - Piracicaba - SP \\ E-mail: passeri@fop.unicamp.br
}

